\title{
Connaissances et attitudes des parents face a la contraception des adolescentes a Yirimadio, Bamako
}

\author{
Knowledge and attitudes of parents towards adolescent contraception in Yirimadio, Bamako
}

Diallo $B^{1 *}$, Kané $A S T^{2}$, Keita $A^{4}$, Sangho $\mathrm{O}^{3,4}$, Koné $\mathrm{M}^{1}$, Sanogo $\mathrm{A}^{5}$, Diallo $\mathrm{A}^{5}$, Coulibaly $\mathrm{B}^{1}, \mathrm{Ba}^{1} \mathrm{~B}$, Touré $\mathrm{K}^{1}$, Diawara $\mathrm{O}^{1}$, Traoré $\mathrm{H}^{1}$, Traoré $\mathrm{Y}^{5}$, Diallo $\mathrm{H}^{6}$, Traoré $\mathrm{A}^{5}$, Nour $\mathrm{MAB}^{7}$, NGatil $\mathrm{M}^{7}$, Keita $\mathrm{H}^{8}$, Koné $\mathrm{MO}^{8}$, Sangho $\mathrm{F}^{3,4}$, Diarra $\mathrm{NH}^{4}$, Coulibaly $\mathrm{CA}^{4,9}$, Kéïta $\mathrm{M}^{10}$, Berthé $\mathrm{M}^{9}$, Telly $\mathrm{N}^{4}$, Kéïta $\mathrm{S}^{4}$, Samaké $\mathrm{A}^{10}$

\section{DOI : 10.53318/msp.v11i1.1886}

${ }^{1}$ Centre Hospitalier Universitaire d'Odontostomatologie CHU-OS 2Direction Centrale des Services de Santé des Armées DCSSA

${ }^{3} \mathrm{D}$ Département d'Enseignement et de Recherche des Sciences Biologiques et Médicales, Faculté de Pharmacie (FAPH), Université des Sciences, des Techniques et des Technologies de Bamako (USTTB) ${ }^{4}$ Département d'Enseignement et de Recherche en Santé Publique (DERSP), Faculté de Médecine et d'Odonto-Stomatologie (FMOS), USTTB

5 Institut National de Formation en Sciences de la Santé

6Ministère de la Promotion de la Femme, de l'Enfant et de la Famille

${ }^{7}$ Centre Hospitalier Universitaire de Référence Nationale de N'Djamena au Tchad

${ }^{8}$ Direction régionale de la santé de Kidal

Institut national de santé publique

${ }^{10} \mathrm{CSREF}$ de la Commune VI de Bamako

*Correspondant : Dr Baba Diallo Hospitalier au CHU CNOS Tel :

74565428, babasidikidiallo@yahoo.fr

\section{RESUME}

Introduction : L'adolescence est une période de transition entre l'enfance et l'âge adulte, au cours de laquelle l'individu acquiert la plénitude de ses fonctions de reproduction où survient une sexualité précoce avec des conséquences comme les grossesses non désirées. L'objectif était d'étudier les déterminants qui influent sur les connaissances et attitudes des parents face à l'utilisation des services de planification familiale (PF) par les adolescentes dans l'aire de santé de Yirimadio. Matériel et méthodes : Nous avons mené une étude transversale descriptive. Elle s'est déroulée pendant 2 mois du 07 décembre 2019 au 07 février 2020. Les parents âgés de plus de 30 ayant acceptés de répondre aux questions étaient inclus dans cette étude. La taille de l'échantillon était de 305. Les données ont été collectées avec l'application Kobo Collecte et analysées sur SPSS version 21. Nous avons obtenu le consentement des répondants. Résultats : Au total $76,06 \%$ des parents associaient la PF à l'espacement des naissances et 7,54\% pensaient que son utilisation avait des inconvénients sur la santé. Environ $92 \%$ ont affirmé son avantage. Environ $36,4 \%$ des parents ont affirmé l'avantage de la PF pour les adolescentes. Pour $66,2 \%$ des parents l'utilisation des services de PF par les adolescentes est mauvaise, $64 \%$ que la PF n'a pas d'avantage sur leur santé et $78,09 \%$ ont affirmé avoir une communication moyenne avec eux. Conclusion : La majorité des parents refusent que leurs adolescentes fréquentent des services de PF. Face à cette situation, il serait important de mettre un accent sur la mobilisation et IEC au sein de la communauté.

Mots clés : connaissance, attitude, parents, PF, adolescente, Yirimadio, Mali

\begin{abstract}
Introduction: Adolescence is a period of transition between childhood and adulthood, during which the individual acquires the fullness of his reproductive functions where precocious sexuality occurs with consequences such as unwanted pregnancies. The objective was to study the determinants that influence parental knowledge and attitudes towards the use of family planning (FP) services by adolescent girls in the Yirimadio health area. Material and methods: We carried out a descriptive cross-sectional study. It took place over 2 months from December 07, 2019 to February 07, 2020. Parents over the age of 30 who agreed to answer the questions were included in this study. The sample size was 305 . The data was collected with the Kobo Collect app and analyzed on SPSS version 21 . We obtained the consent of the respondents. Results: A total of $76.06 \%$ of parents associated FP with birth spacing and 7.54\% believed that its use had health drawbacks. About $92 \%$ claimed its advantage. About $36.4 \%$ of parents affirmed the benefit of FP for adolescent girls. For $66.2 \%$ of parents, the use of FP services by adolescent girls is poor, $64 \%$ that FP has no advantage on their health and $78.09 \%$ said they have average communication with them. Conclusion: The majority of parents refuse to allow their adolescent girls to attend FP services. Faced with this situation, it would be important to put an emphasis on mobilization and IEC within the community.

Keys words: knowledge, attitude, parents, family planning, teenager, Yirimadio, Mali
\end{abstract}

\section{INTRODUCTION}

L'adolescence est une période de transition entre l'enfance et l'âge adulte, au cours de laquelle l'individu acquiert la plénitude de ses fonctions de reproduction. C'est aussi une période pendant laquelle survient une sexualité précoce avec comme résultante des grossesses non désirées, des avortements provoqués clandestins et des maladies sexuellement transmissibles (1). La planification familiale, est l'ensemble des moyens qui concourent au contrôle des naissances, dans le but de permettre aux familles de choisir d'avoir un enfant (2). Selon l'OMS, la Planification Familiale (PF) est l'ensemble de mesures et de méthodes que les hommes et les femmes utilisent pour éviter les grossesses non désirées et préparer la venue des enfants quand ils le désirent $(3,4)$. Les grossesses trop précoces, trop tardives, trop nombreuses, trop rapprochées sont responsables de la majorité de complications obstétricales 
directes, causes de plus de $70 \%$ des décès maternels dans les pays à faibles revenus (3).

En Afrique, la prévalence contraceptive est passée de $23,6 \%$ à $27,6 \%$ respectivement en 2008 et 2014 (5). Elle est estimée à $37 \%$ en 2019 (6). L'utilisation de la contraception par les hommes représente un sous ensemble relativement faible des taux de prévalence cidessus (5).

Selon Noumbissi A. et Sanderson J.P. au Cameroun en 1999, on retrouve une mésentente dans le foyer au sujet du planning familial dans $18 \%$ des cas (7). La femme veut utiliser la contraception et le mari y est farouchement opposé (7). Seule la femme à long terme peut lui faire changer d'avis. Les couples sont généralement favorables à la venue du nouveau bébé (7).

Sidibé I. a mené une étude sur les connaissances, attitudes et pratiques des ménages de la commune urbaine de Yanfolila en matière de PF en 2015 (8). Cette étude montre que parmi les personnes enquêtées $64,6 \%$ connaissaient l'existence des dangers liés aux relations sexuelles précoces contre $35,4 \%$ qui ne les connaissaient pas (8). Ces dangers sont entre autre grossesse précoce, grossesse non désirées, perte de virginité, échec scolaire, IST, condamnation religieuse, hémorragies (8). L'insuffisance d'information sur les effets secondaires des produits contraceptifs, leur rupture périodique et le coût élevé de certaines méthodes constituent les principales raisons de la faible utilisation des services de PF (8).

Kouma M. au Lycée Mamadou Sarr de Bamako en 2008 trouve que $84,61 \%$ des jeunes du lycée avaient un partenaire sexuel (9). Sangho et al. trouvent que $47,2 \%$ des enquêtés avaient un partenaire sexuel dans les lycées de Niono en 2017 (10). L'âge minimum du premier rapport sexuel était de 13 ans ; $35,35 \%$ des élèves discutaient en général de la sexualité avec leur partenaire ; $87,14 \%$ des élèves avaient eu 2 partenaires (9). Parmi les élèves ayant des rapports sexuels avec un partenaire occasionnel, plus de la moitié n'avait pas utilisé de préservatif à Bamako et $27,1 \%$ à Niono $(9,10)$. Tout ceci fait que les adolescentes sont exposées au risque de grossesses précoces et non désirées, aux Infections Sexuellement Transmissibles (IST). Elles sont aussi vulnérables face aux dangers liés à la consommation de tabac, d'alcool et autres drogues. Beaucoup d'entre elles sont confrontées quotidiennement à la violence et à la peur.

La prévalence contraceptive chez les adolescentes est de $9 \%$ (11). Pour cette raison, le niveau de leur fécondité occupe une place importante dans l'élaboration des politiques et la mise en œuvre des stratégies et des programmes de la santé de la reproduction. Par ailleurs, il y a un faible taux d'utilisation des services de PF dans la commune $\mathrm{VI}$ avec seulement $20,7 \%$. C'est ce qui nous a motivés à réfléchir sur l'attitude des parents face à la planification familiale des adolescentes. Les éléments de cette réflexion sont matérialisés dans le cadre conceptuel (Figure 1). L'objectif était d'étudier les déterminants qui influent sur les connaissances et les attitudes des parents face à l'utilisation des services de la planification familiale par les adolescentes dans l'aire de santé de Yirimadio en 2019.

\section{MATERIEL ET METHODES}

Type de l'étude

Nous avons mené une étude transversale descriptive dans l'aire de santé de Yirimadio dans de la commune VI du district de Bamako.

\section{Période d'étude}

Elle s'est déroulée pendant une période de 2 mois du 07 Décembre 2019 au 07 Février 2020.

Population d'étude :

La population d'étude était constituée par les parents âgés d'au moins 30 ans résidant dans l'aire de santé de Yirimadio.

\section{Critères d'inclusion}

$\checkmark \quad$ Etre âgés d'au moins de 30 ans ;

$\checkmark$ Accepter de participer ou de répondre aux questions ;

$\checkmark$ Tous les parents qui vivent dans l'air de santé de Yirimadio.

\section{Critères de non-inclusion}

Les individus dans l'urgence ou la contrainte de temps.

Taille d'échantillon

La taille d'échantillonnage a été déterminée avec la formule de la taille de $D$. Schwartz $\left(n=Z^{2} p q / i^{2}\right)(12)$. Elle a été estimée à 305 personnes.

\section{Echantillonnage}

Les personnes interrogées ont été sélectionnées dans le quartier. La méthode d'échantillonnage probabiliste avec le sondage à 3 degrés (secteur, ménage, individu) a été utilisée pour mener cette étude. Cette technique permettait de donner la même chance à tout individu d'être sélectionné (équiprobabilité). Nous avons sélectionné les secteurs en numérotant chaque secteur. Le quartier a été divisé en 27 secteurs. Nous avons ensuite tiré au hasard 6 secteurs par sondage aléatoire simple. Ne disposant pas la liste de tous les ménages, nous avons choisis 51 ménages par secteur à partir de la maison du chef de quartier. Les individus ont sélectionné dans les ménages jusqu'à l'obtention de l'échantillon.

Ensuite nous avons procédé aux choix des ménages en partant un sur trois pour les secteurs ayant une forte densité populationnelle et un sur deux pour ceux ayant une faible densité en habitant jusqu'à l'obtention de l'échantillon.

\section{Outil et technique de collecte des données :}

La fiche d'entretien individuel a été utilisée comme outil de collecte en mode face à face.

\section{Saisie et analyse des données}

Après le dépouillement des questionnaires, les données ont été saisies sur le logiciel Kobo Collecte. L'analyse a été effectuée avec le logiciel SPSS version 21.

L'acceptabilité a été catégorisée en 3 niveaux à savoir : Niveau 1 d'acceptabilité : Les parents n'acceptent pas que les adolescentes fréquentent le service ; Niveau 2 d'acceptabilité : Les parents acceptent que les adolescentes fréquentent les services pour s'informer sans utiliser les produits contraceptifs; 
- $\quad$ Niveau 3 d'acceptabilité : Les parents qui acceptent que les adolescentes fréquentent les services de PF et peuvent prendre les produits contraceptifs.

\section{Considérations éthiques et déontologiques}

Le but d'étude a été expliqué aux répondants. Nous avons obtenu le consentement éclairé des répondants avant de commencer l'enquête. La confidentialité et l'anonymat des personnes interrogées ont été strictement respectés, la latitude a été donnée à chaque participant qui désirait se retirer au cours de l'enquête, de le faire en toute liberté sans aucune contrainte. L'étude n'apportait aucun préjudice aux participants.

\section{RESULTATS}

Environ $81 \%$ des personnes enquêtées était de sexe féminin avec un sex ratio de 4,3 (Tableau I).La tranche d'âge $45-55$ ans était la plus représentée avec $75,7 \%$ des cas (Tableau I).

Les mariés représentaient plus de la moitié de l'échantillon avec $55,1 \%$ des cas (Tableau I).

Les commerçants (e) étaient les plus représentés avec $42 \%$.

Les parents non scolarisé étaient les plus représentés avec $34,1 \%$ des cas (Tableau I).

La définition de la planification familiale citée par les parents a été l'espacement des naissances avec $76.1 \%$ des cas. Les parents qui ont affirmé connaitre les avantages de la Planification Familiale représentaient 92,5\% des cas (Tableau II).

Les avantages en lien avec l'éviction des grossesses non désirées ont représenté $64,9 \%$ des cas (Tableau III).

Les parents qui ont pensé que la PF avait des inconvénients ont représenté $7,5 \%$ des parents.

Les troubles menstruels ont représenté $65.2 \%$ des inconvénients signalés par les parents (Tableau IV).

Les parents qui ont défini l'adolescence comme étant la période comprise entre 11 ans jusqu'à l'âge du mariage représentaient $41 \%$ des cas.

Les $66,2 \%$ des parents qui pensaient que l'utilisation de la PF était mauvaise pour les adolescents (Tableau V).

Environ $64 \%$ des parents n'acceptaient pas l'utilisation de la PF par leurs adolescentes.

Les parents qui pensaient qu'il y a une différence entre utiliser les produits contraceptifs et fréquenter le service de PF étaient de $51,1 \%$. Environ $63 \%$ des parents pensaient que la fréquentation des services de PF par les adolescentes montre qu'elles sont mal éduquées. Les $69,2 \%$ des parents ont pensé que l'utilisation des produits contraceptifs pourrait engendrer l'infertilité chez les adolescentes. Le niveau de communication des parents avec leurs adolescentes étaient bonne, moyenne et médiocre avec respectivement $28,9 \%, 48,5 \%$ et $22,6 \%$. La fréquence des niveaux d'acceptabilité 1,2 et 3 des parents étaient respectivement de $61 \%, 32 \%$ et $7 \%$.

Environ $56 \%$ des parents pensaient que l'utilisation des services de PF par les adolescentes pourrait favoriser la prostitution (Tableau VI).
Environ $63.6 \%$ des parents ont affirmé que l'utilisation des produits contraceptifs n'a pas d'avantages pour la santé de l'adolescente ainsi que celle de la communauté. Les parents qui ont affirmé que l'utilisation des PF pourrait permettre d'éviter les grossesses non désirées représentaient $50,5 \%$ des cas et éviter les avortements provoqués et clandestins $49,5 \%$.

\section{DISCUSSION}

L'âge médian était de de 39 ans pour des extrêmes de 30 et 71 ans. Plus de trois quart des enquêtés avaient entre 45 à 55 ans. Le tiers était non scolarisé. La non scolarisation pourrait avoir un impact négatif sur la sensibilisation car cette frange de la population pourraient s'accrocher à la tradition plus qu'à la médecine moderne. Plus de trois quarts des parents enquêtés associaient la PF à l'espacement des naissances. Ce résultat est comparable à celui obtenu par Sacho D. qui trouve, dans une étude réalisée au Lycée Bah Aminata Diallo, 87,8\% d'enquêtés pour l'espacement des naissances (13).

Dans notre étude, près de deux tiers des parents ont cité l'éviction des grossesses non désirées comme avantage de la PF. Ce résultat diffère celui de Traoré A.I. qui trouve $43,04 \%$ dans son étude sur les déterminants de l'utilisation du service de PF par les femmes en âge de procréer dans la commune VI du District de Bamako (14). Ceci pourrait s'expliquer par le fait que les jeunes commenceraient la sexualité très tôt et pourraient courir un risque d'attraper une grossesse non souhaitée. Le début précoce de la sexualité a été démontré dans les études de Sangho et al. et de Kouma M $(9,10)$.

Seulement un tiers des parents ont affirmé autoriser l'utilisation des services PF par leurs adolescentes. Les deux tiers trouvaient que la PF était une mauvaise chose pour les adolescentes. Ils ont affirmé que la PF des adolescentes pourrait les inciter à la débauche. Et ils estiment que les adolescentes doivent s'abstenir jusqu'au mariage selon les croyances religieuses et culturelles. En fait il s'agit là d'une croyance populaire au Mali où une femme qui utilise la contraception en l'absence d'autorisation parentale ou de son conjoint, est assimilable à une personne qui entretiendrait des relations intimes en cachette. Hors, la loi autorise la femme à décider librement de sa pratique contraceptive (15). Malgré tout, du fait du poids de la tradition et de la religion, les femmes et les adolescents sont souvent obligées d'obtenir cette autorisation avant de pratiquer. Au Togo, dans $4,8 \%$ et $2,8 \%$ des cas, l'enseignant a laissé sa femme décider seule de l'utilisation et du choix de la méthode à adopter (16).

Plus du tiers des parents trouvaient que la PF a des avantages, et la moitié d'entre eux ont signalé l'éviction de la grossesse non désirée et l'avortement provoqué. Sinayoko $T$ trouve dans son étude sur des 221 jeunes ( 15 à 24 ans) que 79,6\% des adolescents avaient déjà un (e) copain/copine (17). En dehors de l'utilisation des préservatifs pour les partenaires occasionnels et mêmes 
réguliers, le risque de grossesse non désiré est toujours présent $(9,10,13,18)$.

Hodonou KAS. et al. en 2002 au Togo trouvent que les raisons de non utilisation des méthodes contraceptives étaient l'abstinence, les effets secondaires, le manque d'information (19). Les élèves étaient sexuellement actifs et l'âge moyen du premier rapport sexuel était de 16,96 ans chez les garçons et 17,20 ans chez les filles. Les obstacles pour avoir accès aux services de planification familiale étaient l'influence des proches et le manque de centre approprié pour les jeunes et adolescents.

Pour améliorer le taux de PF au niveau des adolescentes, il faudra mettre un accent particulier sur la communication entre les parents et enfants et renforcer les séances de sensibilisation au sein de la communauté sur les risques que les adolescentes pourraient courir (les grossesses précoces et non désirées, les Interruptions Volontaires ou Clandestines de Grossesse (IVG), la rupture utérine, la fistule obstétricale, l'abandon de l'école, les figues, les rejets, etc.).

La gratuité des actes dans les services de PF et des produits contraceptifs pourraient contribuer à améliorer le taux du PF chez les adolescentes et la population générale. Cette gratuité est assuré par l'état et ses partenaires dont Marie Stopes Mali (20).

\section{CONCLUSION :}

La majorité des parents refusent que leurs adolescentes fréquente des services de PF. Face à cette situation, il serait important de mettre un accent sur la communication au sein de la communauté pour qu'elle soit mieux informée sur l'importance de la fréquentation des services PF par les adolescentes et sur les risques que les adolescentes pourraient courir (les grossesses précoces, non désirées, les IVG, la rupture utérine, la fistule obstétricale, l'abandon de l'école, etc.) du fait de l'absence des informations sur leur santé sexuelle et reproductive.

\section{REFERENCES}

1. BA M, SANGARE M, MOREIRA P, BAH M, DIADHIOU F. CONNAISSANCE, PRATIQUE ET PERSPECTIVES DE LA CONTRACEPTION CHEZ LES ADOLESCENTES. Médecine Afr Noire. 1999;46(6):300-2.

\section{AUBIN C, BRANCHU C, VIEILLERIBIERE J-L,}

SITRUK P. Les organismes de planification, de conseil et d'éducation familiale : un bilan [Internet]. France: Inspection générale des affaires sociales; 2011 [cité 25 juill 2021] p. 164. Disponible sur: https://www.igas.gouv.fr/spip.php?article213 3. OMS. Etude sur la planification familiale [Internet]. OMS Genève; 2019 [cité 7 nov 2020]. Disponible sur: www.who.int<topic $>$ famil 4. OMS. Contraception [Internet]. [cité 25 juill 2021]. Disponible sur: https://www.who.int/westernpacific/healthtopics/contraception

5. OMS. Santé de l'adolescent [Internet]. OMS Genève; 2015. Disponible sur: https://www.who.int/topics/adolescent_health/fr/ 6. Le Partenariat de Ouagadougou. Le taux de prévalence contraceptive [Internet]. Partenariat de Ouagadougou. 2019 [cité 26 juill 2021]. Disponible sur: https://partenariatouaga.org/le-taux-de-prevalencecontraceptive-a-atteint-37-en-afrique-responsable/ 7. Noumbissi A, Sanderson J-P. La communication entre conjoints sur la planification familiale au Cameroun. Les normes et les stratégies du couple en matière de fécondité. Population. 1999;54(1):131-44.

8. Sidibé I. Connaissances, attitudes et pratiques comportementales des ménages de la Commune Urbaine de Yanfolila en matière de la planification familiale [Internet] [Thèse de Médecine Générale]. [Bamako, Mali]: USTTB; 2015 [cité 26 juill 2021]. Disponible sur: https://www.bibliosante.m//handle/123456789/796 9. Kouma M. Connaissances, attitudes et pratiques des adolescents du lycée Mamadou Sarr relatives à la planification familiale, infections sexuellement transmissibles et SIDA [Internet] [Thèse de Médecine Générale]. [Bamako, Mali]: USTTB; 2008 [cité 25 juill 2021]. Disponible sur:

http://www.keneya.net/fmpos/theses/2008/med/pdf/08M5 44.pdf

10. Sangho O, Telly N, Sogodogo A, Diarra MM, Touré AY, Sangho $F$, et al. Connaissances, attitudes et pratiques des jeunes lycéens de Niono sur les IST, le $\mathrm{VIH}$ et le SIDA. Rev Malienne Infect Microbiol. 2 juin 2021;16(2):13-9.

11. Institut National de la Statistique (INSTAT), Cellule de Planification et de Statistique Secteur SantéDéveloppement Social et Promotion de la Famille (CPS/SS-DS-PF), ICF. Sixième Enquête Démographique et de Santé au Mali 2018. Indicateurs Clés. [Internet]. Bamako, Mali, et Rockville, Maryland, USA: INSTAT, CPS/SS-DS-PF, et ICF; 2019 [cité 21 mai 2019].

Disponible sur: https://dhsprogram.com/pubs/pdf/PR115/PR115.pdf 12. Ancelle T. Statistique Epidémiologie. 1ère éd. Paris: Maloine; 2002.

13. Sacho D. Connaissances et pratiques des adolescents du lycée Aminata Bah Diallo en matière de la PF, MST et SIDA [Thèse de Médecine Générale]. [Bamako, Mali]: USTTB; 2002. 
14. Traoré A. Déterminant de l'utilisation des services de planification familiale par les femmes en âge de procréer dans le CSRéf de la commune VI du district de Bamako [Internet] [Thèse de Médecine Générale] [Bamako, Mali]: USTTB; 2010 [cité 25 juill 2021]. Disponible sur:

https://www.bibliosante.ml/bitstream/handle/123456789/4 520/20M88.pdf?sequence=1\&isAllowed=y

15. Assemblée Nationale, République du Mali. Loi $n^{\circ} 02-044$ / du 24 juin 2002 Relative à la santé de la reproduction [Internet]. 02 - 044 2002. Disponible sur: https://scorecard.prb.org/wp-

content/uploads/2018/05/Loi-n\%C2\%B0-02-044-Relativea\%CC\%80-la-Sante\%CC\%81-de-la-Reproduction-2002.Mali.pdf

16. HODONOU KAS, ADJAHOTO EO, KODO K, AKPADZA K, BAETA S. CONNAISSANCES, ATTITUDES ET PRATIQUES DES ENSEIGNANTS MARIES DE LA COMMUNE DE LOME (TOGO) EN MATIERE DE CONTRACEPTION. Médecine Afr Noire 199946 89. 1999;46(8/9):410-5.

17. Sinayoko T. connaissances, attitudes et pratiques des jeunes de 15 à 24 ans de la commune l et II du district de Bamako en matière de planification familiale. [Internet] [Thèse de Médecine Générale]. [Bamako, Mali]: USTTB; 2015 [cité 26 juill 2021]. Disponible sur: https://www.bibliosante.ml/handle/123456789/794 18. DJOSSOU TR. Connaissances, attitudes et pratiques des élèves du Lycée Askia Mohamed de la Commune III de Bamako en matière de contraception en 2019 [Internet] [Thèse de Pharmacie]. [Bamako, Mali]: USTTB; 2020 [cité 1 juill 2021]. Disponible sur: https://www.bibliosante.ml/bitstream/handle/123456789/3 973/20P47.pdf?sequence=1\&isAllowed=y 19. Ajavon DRD, Bassowa A, Logbo-Akey KE, Aboubakari AS, Adom E, Potcho E, et al. Connaissances, attitudes, pratiques des methodes contraceptives dans les lycees publics de la ville de Kara (Togo). J Rech Sci L’Université Lomé. 2018;20(3):365-73.

20. Marie Stopes Mali. Marie Stopes Mali [Internet]. [cité 28 juill 2021]. Disponible sur: https://www.mariestopes.ml/
Liste des tableaux et figures

Tableau II : répartition des parents selon leur définition de la PF.

\begin{tabular}{lcc}
\hline \hline Définition de la PF & $\mathbf{n}$ & $\%$ \\
\hline \hline Espacement des naissances & 232 & 76,1 \\
Limitation des naissances & 12 & 3,9 \\
Eviction des grossesses & 61 & 20,0 \\
Total & $\mathbf{3 0 5}$ & $\mathbf{1 0 0}$ \\
\hline \hline
\end{tabular}

Tableau III : Répartition des parents selon les avantages les plus connus.

\begin{tabular}{lcc}
\hline \hline Avantages de la PF & $\mathbf{n}$ & $\%$ \\
\hline \hline Epanouissement de la famille & 68 & 24,1 \\
Diminution des dépenses familiales & 31 & 11,0 \\
Eviction des grossesses non & 183 & 64,9 \\
désirées & & \\
Total & $\mathbf{2 8 2}$ & $\mathbf{1 0 0}$ \\
\hline \hline
\end{tabular}

Tableau IV : Répartition des parents selon les inconvénients les plus connus.

\begin{tabular}{lcc}
\hline \hline Inconvénients de la PF & $\mathbf{n}$ & $\%$ \\
\hline \hline Infertilité & 5 & 21,8 \\
Troubles menstruels & 15 & 65,2 \\
Autres & 3 & 13,0 \\
Total & 23 & 100 \\
\hline \hline
\end{tabular}

Tableau V : Répartition des parents selon leur degré d'appréciation par rapport à l'utilisation des services de PF par les adolescentes.

\begin{tabular}{lcc}
\hline Appréciation de l'utilisation de la & $\mathbf{n}$ & $\%$ \\
PF par les Adolescentes & & \\
\hline \hline Mauvais & 202 & 66,2 \\
Bon & 87 & 28,5 \\
Très Bon & 16 & 5,3 \\
Total & 305 & 100 \\
\hline \hline
\end{tabular}

Tableau VI: Répartition des parents en fonction des points de vue de la communauté

\begin{tabular}{lcc}
\hline \hline $\begin{array}{l}\text { Points de vue de la communauté } \\
\text { sur l'utilisation des services PF }\end{array}$ & $\mathbf{n}$ & $\%$ \\
\hline \hline Acceptée par la société & 21 & 6,9 \\
Favorise la prostitution & 171 & 56,0 \\
Favorise les débauches & 71 & 23,3 \\
Autres & 42 & 13,8 \\
Total & $\mathbf{3 0 5}$ & $\mathbf{1 0 0}$ \\
\hline \hline
\end{tabular}


Tableau I : Répartition des parents selon les caractéristiques socio-démographiques

\begin{tabular}{lccc}
\hline \hline \multicolumn{2}{l}{ Caractéristiques sociodémographiques } & $\mathbf{n}$ & $\%$ \\
\hline \hline \multirow{2}{*}{ Féminin } & Sexe & 305 & 100 \\
Masculin & & 248 & 81,3 \\
& Age & 57 & 18,7 \\
$30-45$ ans & 305 & 100 \\
$45-55$ ans & 5 & 1,7 \\
$55-65$ ans & 231 & 75,7 \\
$>66$ ans & 57 & 18,7 \\
& Statut matrimonial & 12 & 3,9 \\
Marié(e) & 305 & 100 \\
Célibataire & 168 & 55,1 \\
Veuf (ve) & 25 & 8,2 \\
Divorcé(e) & Niveau d'instruction & 70 & 22,9 \\
& 42 & 13,8 \\
Non scolarisé & 305 & 100 \\
Niveau Primaire & 104 & 34,1 \\
Niveau Secondaire & 77 & 25,2 \\
Ecole coranique & 29 & 9,5 \\
Niveau Supérieur & 46 & 15,1 \\
\hline
\end{tabular}

Cadre conceptuel de la planification familiale des adolescentes

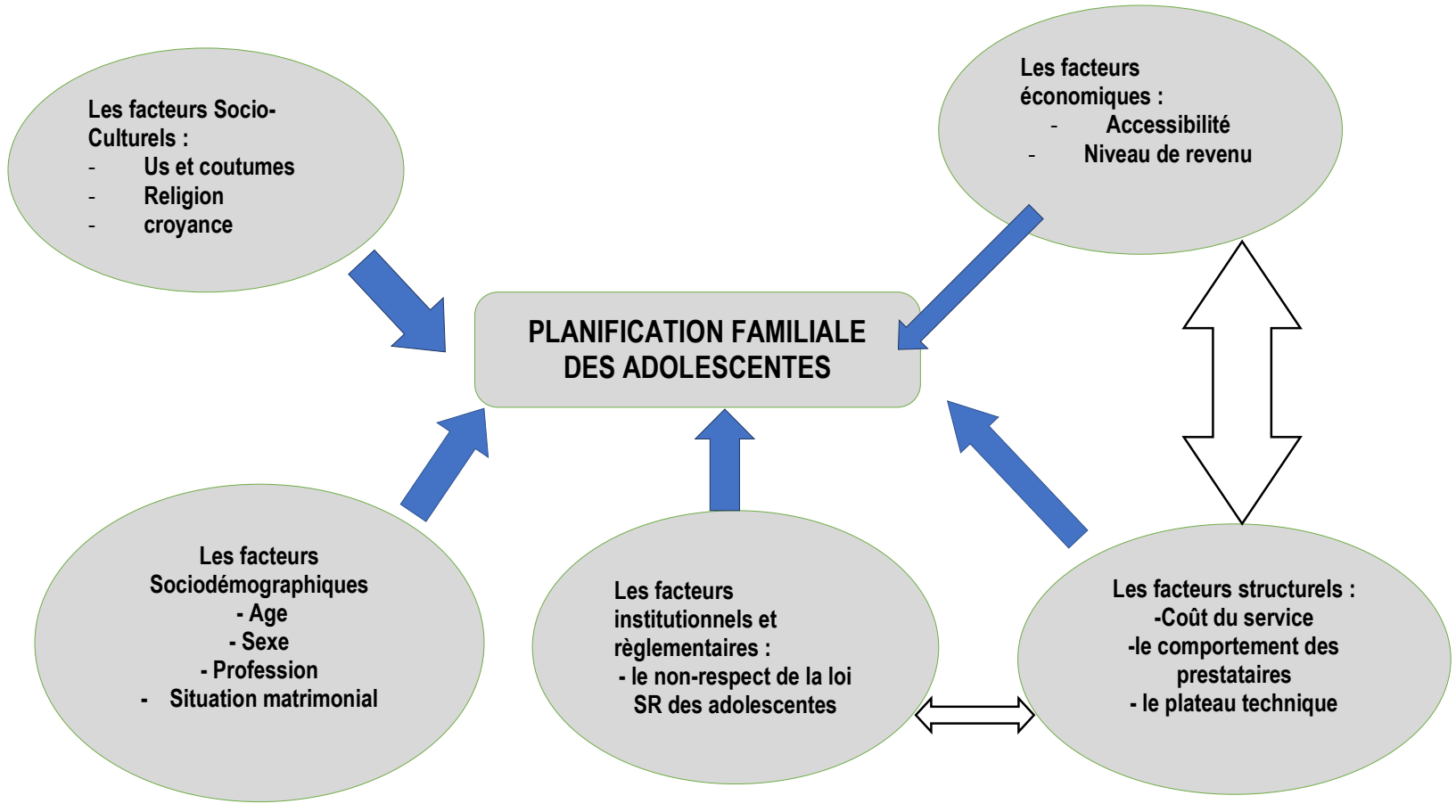

figure 1: Cadre conceptuel sur la connaissance et l'attitude des parents face a l'utilisation des services de PF par les adolescents. 